BMJ Open Sport \& Exercise Medicine

\title{
Paradox of hypercholesterolaemia in highly trained, keto-adapted athletes
}

\author{
Brent C Creighton, ${ }^{1}$ Parker Neil Hyde, ${ }^{2}$ Carl M Maresh,, ${ }^{2}$ William J Kraemer, ${ }^{2}$ \\ Stephen D Phinney, ${ }^{1}$ Jeff S Volek ${ }^{2}$
}

To cite: Creighton BC, Hyde PN, Maresh CM, et al. Paradox of hypercholesterolaemia in highly trained, ketoadapted athletes. BMJ Open Sport \& Exercise Medicine 2018;4:e000429. doi:10.1136/ bmjsem-2018-000429

Accepted 5 September 2018
Check for updates

(C) Author(s) (or their employer(s)) 2018. Re-use permitted under CC BY-NC. No commercial re-use. See rights and permissions. Published by BMJ.

${ }^{1}$ Virta Health, San Francisco, California, USA

${ }^{2}$ Department of Human Sciences, The Ohio State University, Columbus, Ohio, USA

Correspondence to Dr Jeff S Volek; jvolek7@gmail. com

\section{ABSTRACT}

Objective A growing number of ultra-endurance athletes have switched to a very low-carbohydrate/high-fat eating pattern. We compared markers of cholesterol and the lipoprotein profile in a group of elite ultra-runners consuming a high-carbohydrate $(\mathrm{HC})$ or low-carbohydrate (LC) diet.

Methods Fasting blood was obtained from competitive male ultra-endurance runners habitually consuming a very low-carbohydrate $(\mathrm{LC} ; \mathrm{n}=10)$ or high-carbohydrate $(H C ; n=10)$ diet to determine blood cholesterol profile, lipoprotein particle distribution and sterol biomarkers of cholesterol balance.

Results Plasma total cholesterol, low-density lipoprotein (LDL-C) and high-density lipoprotein (HDL-C) cholesterol were all significantly greater $(\mathrm{p}<0.000)$ in the $\mathrm{LC}$ group (65\%, $83 \%$ and $60 \%$, respectively). There were also significant differences in lipoprotein particle distribution as evidenced by a greater size and concentration of large HDL and LDL particles, and total LDL particle concentration was significantly greater in the LC group, but they had significantly fewer small LDL particles.

Conclusion Ultra-endurance athletes habitually consuming a very low-carbohydrate/high-fat diet for over a year showed unique cholesterol profiles characterised by consistently higher plasma LDL-C and HDL-C, less small LDL particles, and lipoprotein profiles consistent with higher insulin sensitivity. There may be a functional purpose to the expansion of the circulating cholesterol pool to meet the heightened demand for lipid transport in highly trained, keto-adapted athletes.

\section{INTRODUCTION}

Ketogenic diets are effective in the management of obesity, ${ }^{1}$ metabolic syndrome ${ }^{2}$ and type 2 diabetes. ${ }^{3}$ Athletes, especially those in the ultra-endurance community, have increasingly embraced lower carbohydrate/higher fat eating patterns. ${ }^{45}$ Whereas carbohydrate restriction and nutritional ketosis consistently improve dyslipidaemia (high triglycerides, low high-density lipoprotein-cholesterol (HDL-C) and predominance of small low-density lipoprotein (LDL) particles) ${ }^{2}$ the effects on total and LDL-cholesterol (LDL-C) are less predictable. Based on our prior ketogenic diet intervention studies in both sedentary and recreationally active men and women with a wide range of
Summary Box

This study showed that a group of elite athletes habitually consuming a very low-carbohydrate (LC) diet for over a year exhibited markedly elevated concentrations of total and LDL-C, above levels considered desirable and beyond that which has been observed in ketogenic diet interventions in non-athletes.

- The LC athletes also had extremely high concentrations of HDL-C and fewer small, dense LDL particles, suggestive of lower risk for cardiovascular disease.

- The explanation for this paradox of very high circulating cholesterol in highly-trained endurance athletes who adopt a low-carbohydrate diet, may be related to high intakes of saturated fat and cholesterol as well as an increased demand for lipid metabolism and corresponding expansion of the intravascular cholesterol pool to accommodate their dramatically accelerated rates of fatty acid oxidation.

adiposity and insulin resistance, ${ }^{6-11}$ serum LDL-C responses varied widely in magnitude and direction, with the mean response often not significantly different from baseline.

Regular aerobic exercise consistently increases HDL-C, specifically the larger HDL2-C fraction, with more robust effects observed with higher levels of training. ${ }^{12} 13$ Aerobic exercise also decreases LDL-C, smaller LDL particles and triglycerides, ${ }^{14}$ but the effects are variable and are more consistent with higher volumes of exercise. ${ }^{15}$ Studies in ultra-endurance athletes indicate they have significantly lower total and LDL-C, and higher HDL-C concentrations, compared with sedentary individuals. ${ }^{1617}$

Knowledge of how ketogenic diets interact with high levels of exercise training to impact cholesterol/lipoprotein profiles is limited. Elite cyclists fed a 4-week eucaloric ketogenic diet during training increased total cholesterol from $156 \mathrm{mg} / \mathrm{dL}$ to $208 \mathrm{mg} / \mathrm{dL}{ }^{18} \mathrm{It}$ remains unclear if serum cholesterol would have continued to rise if the ketogenic diet was extended. Male monozygotic twins with vastly disparate habitual levels of physical activity who consumed both a low-fat/high-carbohydrate diet and a moderate-fat/moderate-carbohydrate diet for 6 weeks showed the expected 
decrease in HDL-C and size of LDL particles with high-carbohydrate intake, with a high degree of concordance within twin pairs. ${ }^{19}$ This result suggests that extreme differences in exercise have little impact on serum lipoprotein responses to diets varying in carbohydrate and fat.

To study whether chronic high levels of exercise training would be associated with attenuation or accentuation of the 'normal' cholesterol and lipoprotein responses associated with a ketogenic diet, we measured fasting serum cholesterol and lipoprotein profiles in ultra-endurance athletes who had been habitually consuming a very low-carbohydrate/high-fat diet for $>6$ months compared with a matched group of athletes consuming a high-carbohydrate diet.

\section{METHODS}

\section{Experimental approach}

The following data are an extension of a larger cross-sectional investigation ${ }^{4}$ that reported on metabolic responses in two groups of elite ultra-marathoners habitually consuming either a low-carbohydrate (LC) or high-carbohydrate (HC) diet. The purpose of the current analysis was to more closely examine differences in cholesterol and lipoprotein markers between these groups.

\section{Study participants}

Twenty highly trained, male ultra-endurance runners consuming an $\mathrm{LC}(\mathrm{n}=10)$ or an $\mathrm{HC}(\mathrm{n}=10)$ diet $21-45$ years of age were selected for participation. Athletes competed in sanctioned running events $\geq 50 \mathrm{~km}$ and/or triathlons of at least half ironman distance $(113 \mathrm{~km})$ and were in the top $10 \%$ of finalists. Athletes were matched for age, physical characteristics, primary competition distance and competition times. All but one athlete travelled via plane to our laboratory for 2 days of testing. Interested athletes spoke extensively with a registered dietitian about their diet history, and completed questionnaires to assess their diet, training, running competition and medical history. At least one phone call was scheduled to review this information and determine eligibility and availability. Diet information was entered into nutrient analysis software (Nutritionist Pro, Axxya Systems, Stafford, Texas). Subjects had to be consuming an LC $(<20 \%$ en carbohydrate, $>60 \%$ en fat) for $>6$ months or $>55 \%$ en from carbohydrate for the HC group. Athletes were excluded if they had diabetes, heart disease, kidney, liver, or other metabolic or endocrine dysfunction, current injury, or anti-inflammatory medication use. Subjects were informed of the purpose and possible risks of the investigation prior to signing an informed consent document.

\section{Measurements}

Full methods and metabolic results have been published. ${ }^{4}$ Athletes arrived at the laboratory at 06:00 after a 10-hour overnight fast and were asked to restrict caffeine, over-thecounter medications and alcohol. The night prior and the morning before testing, subjects were encouraged to liberally consume water to ensure hydration. A urine sample was provided to assess specific gravity (Model A300CL, Spartan, Japan). All subjects had a urine specific gravity (USG) $>1.025$, indicating adequate hydration. Body composition was determined by dual-energy X-ray absorptiometry (Prodigy, Lunar, Madison, Wisconsin). Body weight was recorded to the nearest $0.1 \mathrm{~kg}$ on a digital scale (OHAUS, Florham Park, New Jersey).

\section{Blood collection and analysis}

All blood samples were obtained with a $21 \mathrm{G}$ butterfly needle from an antecubital vein of the subject. After resting quietly for $15 \mathrm{~min}$ in a supine position, blood was collected into EDTA and serum separator vacutainer tubes (Vacuette, Greiner Bio-One North America, Monroe, North Carolina). EDTA tubes were immediately spun while serum tubes remained at room temperature for 15 min prior to centrifugation to allow clotting to occur. Whole blood was centrifuged $\left(1500 \times g\right.$ for $15 \mathrm{~min}$ at $\left.4^{\circ} \mathrm{C}\right)$, promptly aliquoted into cryostorage tubes, snap-frozen with liquid nitrogen and stored at $-80^{\circ} \mathrm{C}$ for later analysis. Frozen samples were thawed only once before the analysis of all variables.

HDL-C and triglycerides were analysed using enzymatic methods on an automated analyser (cobas C 111, Roche Diagnostics, Indianapolis, Indiana). Total cholesterol was measured using enzymatic colourimetric methods. ${ }^{20}$ Plasma LDL-C was determined using a direct method, ${ }^{21}$ as well as calculated from the total cholesterol, HDL-C and triglyceride concentrations. ${ }^{22}$

Lipoprotein distribution was conducted by Liposcience (Raleigh, North Carolina) using hydrogen nuclear magnetic resonance (NMR) on a $400 \mathrm{MHz}$ NMR analyser (Bruker BioSpin, Billerica, Massachusetts) as previously described. ${ }^{2324}$ Lipoprotein subclasses were grouped based on particle diameters: large very low-density lipoprotein (VLDL) (>60 nm), medium VLDL (35-60 nm), small VLDL (27-35 nm), intermediate-density lipoproteins (IDL) (23-27 nm), large LDL (21.2-23 nm), medium LDL (19.8 $21.2 \mathrm{~nm})$, small LDL (18-19.8 nm), large HDL (8.8-13 $\mathrm{nm})$, medium HDL (8.2-8.8 $\mathrm{nm}$ ) and small HDL (7.3-8.2 $\mathrm{nm})$. The lipoprotein insulin resistance index (LP-IR) was calculated from six lipoprotein measures (VLDL, LDL and HDL size, large VLDL particles, small LDL particles and large HDL particles). ${ }^{25}$

Concentrations of non-cholesterol sterol precursors (lathosterol, desmosterol, cholestanol, sitosterol and campesterol) were quantified by Boston Heart Diagnostics using a gas chromatography-mass spectrometry method. ${ }^{26}$ Data are presented in absolute concentration and normalised to total cholesterol $(\mu \mathrm{mol} / \mathrm{mmol}$ of cholesterol) since the non-cholesterol sterol precursors are transported in plasma by lipoproteins. Fractional cholesterol balance was calculated using the following formula: (fractional cholesterol balance $=($ lathosterol $\times 0.8+$ desmosterol $\times 0.2) /($ beta-sitosterol $\times 0.5+$ campesterol $\times 0.5)$ ).

\section{Statistical analysis}

Means and SD were calculated for all variables. Differences between groups were assessed using independent 
Table 1 Baseline subject demographics and habitual daily nutrient intake*

\begin{tabular}{|c|c|c|c|c|c|c|}
\hline & \multicolumn{2}{|c|}{ High-carbohydrate } & \multicolumn{2}{|c|}{ Low-carbohydrate } & \multirow[b]{2}{*}{$P$ values } & \multirow[b]{2}{*}{ Effect size } \\
\hline & Mean & SD & Mean & SD & & \\
\hline Age (years) & 32.9 & 6.0 & 34.1 & 7.1 & 0.689 & 0.18 \\
\hline Height (cm) & 173.9 & 5.3 & 175.7 & 6.5 & 0.555 & 0.30 \\
\hline Body mass (kg) & 66.5 & 6.8 & 68.8 & 8.2 & 0.491 & 0.31 \\
\hline Body mass index $\left(\mathrm{kg} / \mathrm{m}^{2}\right)$ & 22.0 & 1.9 & 22.2 & 0.9 & 0.73 & 0.13 \\
\hline Body fat $(\%) \dagger$ & 9.6 & 4.3 & 7.8 & 2.4 & 0.266 & 0.52 \\
\hline Lean mass (kg) & 57.3 & 5.0 & 60.9 & 7.1 & 0.387 & 0.59 \\
\hline Fat mass (g) & 6.5 & 3.6 & 5.5 & 1.8 & 0.207 & 0.35 \\
\hline $\mathrm{VO}_{2} \max (\mathrm{mL} / \mathrm{kg} / \mathrm{min})$ & 64.3 & 6.2 & 64.7 & 3.7 & 0.299 & 0.08 \\
\hline $\mathrm{VO}_{2} \max (\mathrm{L} / \mathrm{min})$ & 4.3 & 0.5 & 4.5 & 0.4 & 0.850 & 0.44 \\
\hline Competitive running experience (years) & 9.0 & 6.0 & 11.0 & 8.0 & 0.583 & 0.28 \\
\hline Energy (kcal/day) $\ddagger$ & 3044 & 604 & 2884 & 814 & 0.380 & 0.22 \\
\hline Protein (g/day) & 117 & 39 & 139 & 32 & 0.186 & 0.62 \\
\hline Protein (\%en) & 14.9 & 3.9 & 19.4 & 2.4 & 0.001 & 1.39 \\
\hline Protein (g/kg) & 1.7 & 0.4 & 2.1 & 0.6 & 0.192 & 0.78 \\
\hline Carbohydrates (g/day) & 442 & 116 & 82 & 62 & 0.000 & 3.87 \\
\hline Carbohydrates (\%en) & 56.5 & 10.2 & 10.4 & 4.9 & 0.000 & 5.76 \\
\hline Fat (g/day) & 94 & 33 & 226 & 66 & 0.000 & 2.53 \\
\hline Fat (\%en) & 26.7 & 7.5 & 69.5 & 6.0 & 0.000 & 6.30 \\
\hline Saturated fat (g/day) & 22 & 10 & 86 & 22 & 0.000 & 3.75 \\
\hline Monounsaturated fat (g/day) & 32 & 16 & 82 & 42 & 0.001 & 1.57 \\
\hline Polyunsaturated fat (g/day) & 17 & 9 & 28 & 17 & 0.106 & 0.81 \\
\hline Alcohol (\%en) & 1.8 & 2.7 & 0.7 & 1.4 & 0.310 & 0.51 \\
\hline Cholesterol (mg/day) & 291 & 256 & 844 & 351 & 0.000 & 1.80 \\
\hline Fibre (g/day) & 48 & 15 & 23 & 17 & 0.000 & 1.56 \\
\hline
\end{tabular}

*Values are mean $\pm S D(n=10)$.

${ }^{\dagger}$ Determined by dual-energy $\mathrm{X}$-ray absorptiometry.

${ }^{\ddagger}$ 3-day 24-hour food record including 1 weekend day.

$\mathrm{VO}_{2}$ max, maximal oxygen consumption.

samples t-tests. Normality testing was performed using the Shapiro-Wilk test. The following non-normal data were analysed using the Mann-Whitney U: triglycerides, LP-IR, HDL particle size, total LDL particles, IDL particles, small LDL particles, total VLDL particles, large VLDL particles and medium VLDL particles. Normal data with significant variance were analysed using the Welch's unequal variance t-test. A priori power calculations via $G^{*}$ Power were conducted for our previously reported primary outcome (fatty acid oxidation rates) ${ }^{4}$ with a statistical power of $80 \%$ and significance of 0.05. Cohen's $d$ effect sizes were calculated. All statistical analyses were preformed using the Prism GraphPad software (V.6.0, La Jolla, California).

\section{RESULTS}

\section{Subject characteristics and diet}

There were no differences between groups in physical characteristics or aerobic capacity, or caloric intake, but the composition of the diet was dramatically different as per the experimental design (table 1). Two athletes in each group were triathletes, and all others competed in events largely ranging from $80 \mathrm{~km}$ to $161 \mathrm{~km}$ (50-100 miles). Athletes consuming an LC diet derived a majority of their energy from fat $(70 \%)$, predominantly in the forms of saturated and monounsaturated fatty acids. Only $\sim 10 \%$ of energy intake was from carbohydrate sources. Conversely, athletes consuming an HC diet consumed over half their energy in the form of carbohydrates $(57 \%)$. Protein was not significantly different between groups. Dietary cholesterol intake was significantly greater $(\mathrm{p}<0.003)$ among the LC athletes $(844$ $\mathrm{mg}$ /day) compared with the HC athletes $(291 \mathrm{mg} /$ day) . The average duration on an LC diet ranged from 9 to 36 months.

\section{Plasma lipids}

Compared with the HC group, athletes consuming an LC diet had greater total cholesterol (65\%), LDL-C (calculated) (79\%), LDL-C (direct) $(83 \%)$ and HDL-C (60\%) (table 2 , figure 1 ). There was a high degree of correlation 
Table 2 Cholesterol and lipids*

\begin{tabular}{|c|c|c|c|c|c|c|}
\hline & \multicolumn{2}{|c|}{ High-carbohydrate } & \multicolumn{2}{|c|}{ Low-carbohydrate } & \multirow[b]{2}{*}{$P$ values } & \multirow[b]{2}{*}{ Effect size } \\
\hline & Mean & SD & Mean & SD & & \\
\hline Total cholesterol (mg/dL) & 169 & 24 & 278 & 51 & 0.000 & 2.73 \\
\hline Direct LDL-C (mg/dL) & 88 & 14 & 161 & 38 & 0.000 & 2.55 \\
\hline Calculated LDL-C (mg/dL) & 91 & 14 & 163 & 38 & 0.000 & 2.51 \\
\hline HDL-C (mg/dL) & 64 & 18 & 102 & 26 & 0.001 & 1.70 \\
\hline Total cholesterol:HDL-C ratio & 2.74 & 0.47 & 2.81 & 0.53 & 0.744 & 0.14 \\
\hline Triglycerides (mg/dL) & 70 & 25 & 63 & 17 & 0.566 & 0.33 \\
\hline Triglyceride:HDL-C ratio & 1.15 & 0.49 & 0.67 & 0.23 & 0.011 & 1.25 \\
\hline Glucose (mg/dL) & 95 & 4 & 96 & 7 & 0.666 & 0.20 \\
\hline Insulin (pmol/L) & 10.4 & 2.5 & 8.1 & 2.6 & 0.066 & 0.91 \\
\hline
\end{tabular}

To convert to SI units, multiply total cholesterol, LDL-C and HDL-C $(\mathrm{mg} / \mathrm{dL}) \times 0.0256=\mathrm{mmol} / \mathrm{L} ;$ multiply triglycerides $(\mathrm{mg} / \mathrm{dL}) \times 0.0113=$ $\mathrm{mmol} / \mathrm{L}$.

*Values are mean $\pm S D(n=10)$.

HDL-C, high-density lipoprotein-cholesterol; LDL-C, low-density lipoprotein-cholesterol.

in LDL-C concentration ( $\mathrm{r}=0.992)$ between the calculated and direct methods of determination. Plasma triglycerides and the total cholesterol/HDL-C ratio were not different between groups. LC athletes had a lower triglyceride (TG):HDL ratio (44\%). There was no difference in fasting glucose and a trend for a lower insulin in LC athletes.

\section{Lipoprotein particles}

LC athletes had smaller VLDL, and larger LDL and HDL particles (table 3, figure 2). LC athletes had a greater concentration of large HDL and LDL and fewer small LDL particles. Total LDL particle concentration was greater in LC athletes. The LC group had greater large HDL particles. The LP-IR was lower in the LC group.

\section{Fractional cholesterol balance and non-cholesterol sterols}

Absolute concentrations of sterols were greater in the LC group for desmosterol and cholestenol (table 4). When normalised to total cholesterol, lathosterol, cholestanol and campesterol were higher and desmosterol was lower in the HC group. The calculated fractional cholesterol balance was not significantly different between groups.

\section{DISCUSSION}

We compared cholesterol profiles in two groups of highly trained, ultra-endurance athletes who were chronically adapted to either an LC or HC diet. All LC athletes had total cholesterol $>200 \mathrm{mg} / \mathrm{dL}$ and LDL-C $>100 \mathrm{mg} /$ $\mathrm{dL}$, whereas in a matched group of HC athletes all but two were under these thresholds considered desirable/ optimal. ${ }^{27}$ Despite the high LDL-C, LC athletes had less small LDL particles, and HDL-C levels were considerably higher than expected in trained athletes. ${ }^{12} 131617$ The exaggerated hypercholesterolaemia exhibited in chronically keto-adapted endurance athletes is counterintuitive in consideration that (1) the cholesterol levels in LC athletes are greater in magnitude than has been reported in non-athletes consuming a ketogenic $\operatorname{diet}^{28}$ (2) ultra-endurance athletes are reported to have similar or lower total cholesterol and LDL-C than less active individuals, ${ }^{16}{ }^{17}$ (3) exercise training studies report either no change or slight reductions in total and LDL-C, ${ }^{15} 29$ (4) previous studies in endurance athletes fed high-fat diets (50\%-85\% of energy) for $2-12$ weeks indicate total cholesterol concentrations remain under or slightly above $200 \mathrm{mg} / \mathrm{dL},{ }^{18} 3031$ and (5) disparate levels of exercise have little impact on the typical cholesterol responses to diets varying in carbohydrate and fat. ${ }^{19} \mathrm{We}$ propose several explanations for the apparent paradox of high-volume exercise training accentuating, as opposed to attenuating, the cholesterolaemic response to a ketogenic diet.

Typical cholesterol profiles associated with ketogenic diets In a review of 15 low-fat diet comparison studies, ${ }^{28}$ it was reported that in all cases, a very low-carbohydrate diet led to greater increases in total cholesterol, LDL-C and HDL-C. The mean difference between the relative changes for each diet was $7 \%$ for total cholesterol, $9 \%$ for LDL-C and $11 \%$ for HDL-C. The highest mean increase in LDL-C for any study reviewed was $15 \% .^{8}$ By comparison, total cholesterol, LDL-C and HDL-C were $64 \%, 79 \%$ and $59 \%$ higher in $\mathrm{LC}$ athletes relative to their HC counterparts. Despite the nearly twofold higher LDL-C concentrations in LC athletes, small LDL particle concentration was $56 \%$ lower than $\mathrm{HC}$ athletes. The shift from small to large LDL particles is independent of the change in LDL-C and consistent with the strong correlation between dietary carbohydrate and LDL size ${ }^{32}$ and is in agreement with many other ketogenic diet interventions. $^{71}{ }^{33}$ High HDL-C and large HDL2-C in LC athletes were expected, but the magnitude of difference is noteworthy. All $10 \mathrm{LC}$ athletes had HDL-C higher than the mean HDL-C level in $\mathrm{HC}$ athletes $(64 \mathrm{mg} / \mathrm{dL})$. A 


\section{- High Carb \\ - Low Carb}
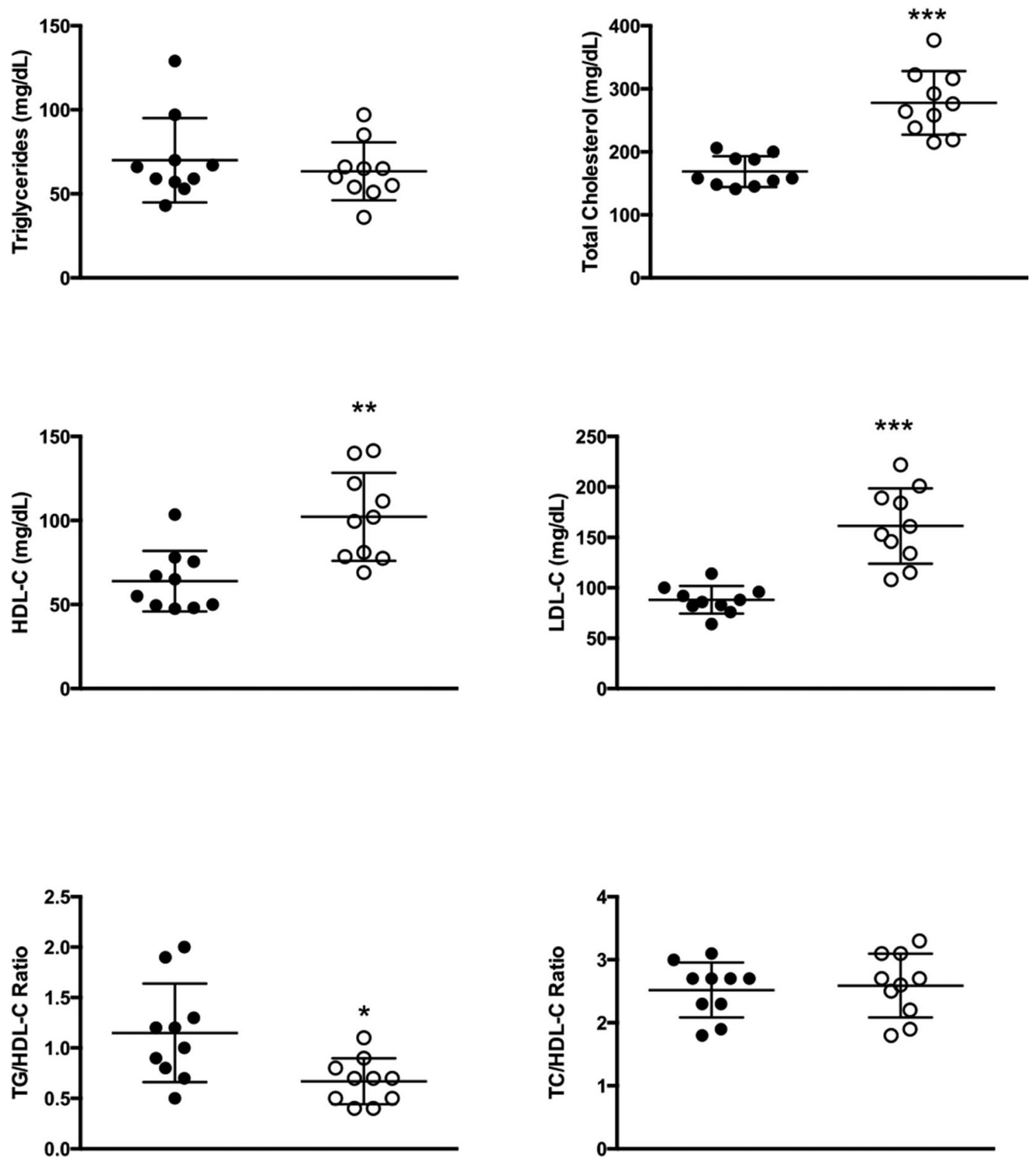

Figure 1 Individual lipid measures for high-carbohydrate $(n=10)$ and low-carbohydrate $(n=10)$ ultra-endurance athletes. Bars represent mean $\pm 1 S D$. HDL-C, high-density lipoprotein-cholesterol; LDL-C, low-density lipoprotein-cholesterol. TC, total cholesterol; TG, triglyceride. ${ }^{*} \mathrm{P}<0.05 ;{ }^{* *} \mathrm{P}<0.01 ;{ }^{* * *} \mathrm{P}<0.001$.

decrease in serum triglycerides is a hallmark response to a ketogenic diet, but both groups had similar low levels of triglycerides, suggesting that additional mechanism(s) beyond those related to TG lowering account for the differences in HDL-C.

\section{Reasons for hypercholesterolaemic profiles in LC athletes} The hypercholesterolaemia observed in LC athletes could be partially explained by dietary factors including greater intake of saturated fat ( $86 \mathrm{vs} 21 \mathrm{~g} /$ day) and cholesterol
( 844 vs $251 \mathrm{mg} /$ day), and lower intake of fibre (23 vs 57 $\mathrm{g} /$ day). Meta-analyses indicate that higher intake of saturated $\mathrm{fat}^{34}$ and cholesterol ${ }^{35}$ and lower intake of fibre ${ }^{36}$ are associated with increased blood cholesterol. However, the predicted increase in blood cholesterol from these dietary factors, even if viewed collectively, falls short of explaining the significantly higher blood cholesterol levels in LC athletes. We observed significant associations between dietary cholesterol, saturated fat and fibre intake 
Table 3 HDL, LDL and VLDL size and concentration of total particles and subfractions*

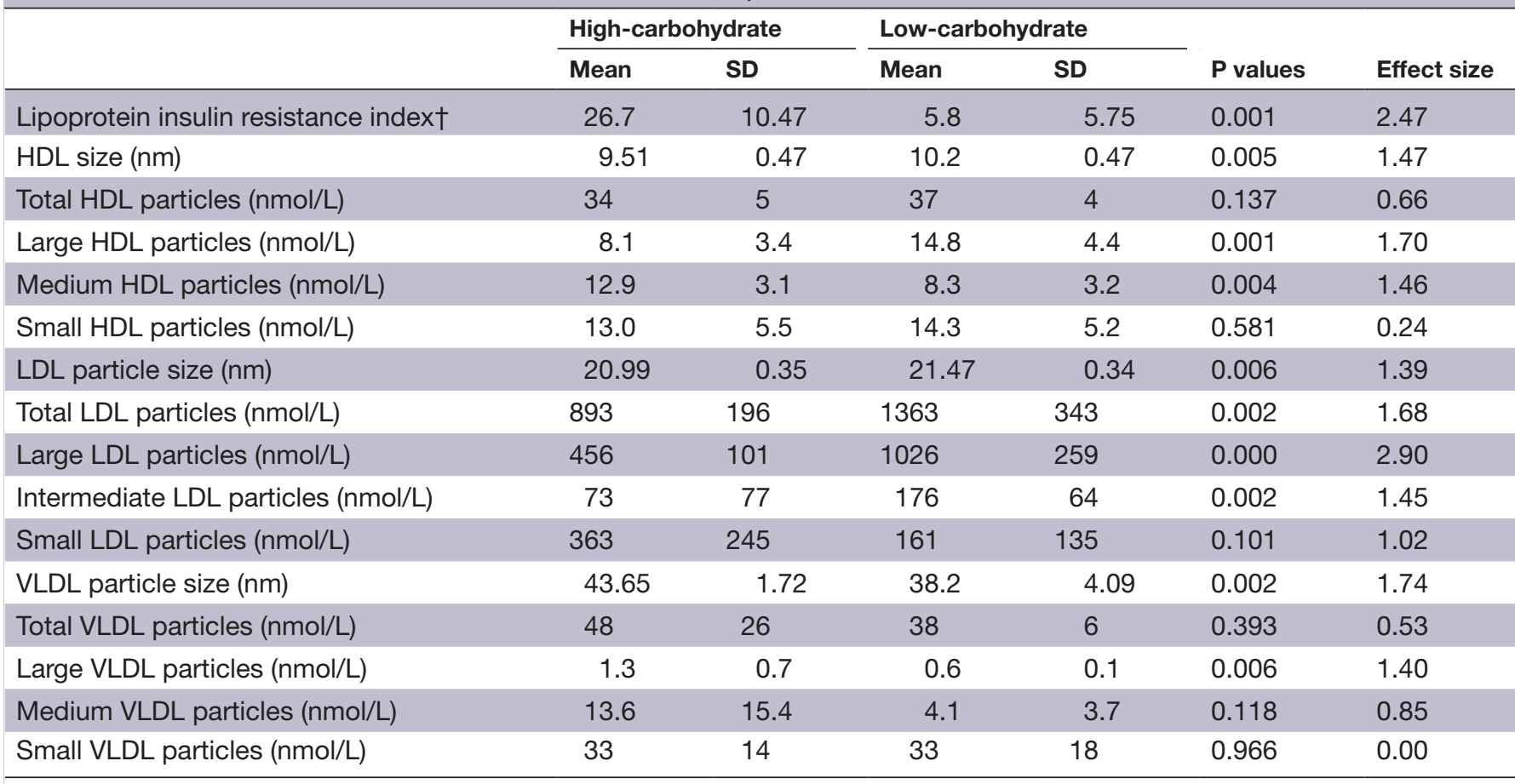

*Values are mean $\pm S D(n=10)$.

†Combined algorithm of six lipoprotein measures.

HDL, high-density lipoprotein; LDL, low-density lipoprotein.

with blood cholesterol measures, but their individual role is impossible to ascertain from correlations and the high degree of inter-relation among dietary nutrients.

Several biological processes involved in cholesterol homeostasis may be altered in keto-adapted athletes that manifest in increased circulating cholesterol. An increase in dietary cholesterol is normally balanced by some combination of decreased exogenous cholesterol absorption, decreased endogenous cholesterol synthesis and increased biliary cholesterol output such that circulating cholesterol levels are not significantly altered. It is noteworthy that ketogenesis and cholesterol synthesis share a common pathway whereby acetyl CoA is converted to acetoacetyl-CoA and then $\beta$-Hydroxy $\beta$-methylglutaryl-CoA (HMG-CoA) by thiolase and HMG-CoA synthase, respectively. ${ }^{37}$ In the synthesis of cholesterol, HMG-CoA is converted to mevalonate by HMG-CoA reductase, whereas in the synthesis of ketones HMG-CoA is converted to acetoacetate by HMG-CoA lyase. Although ketone synthesis occurs exclusively in the mitochondria and cholesterol synthesis in the cytoplasm, ${ }^{37}$ it is possible for some acetoacetate generated during ketogenesis to diffuse out of the mitochondria and be converted to acetoacetyl-CoA in the cytosol via the action of acetoacetyl-CoA synthetase. ${ }^{38}$ Thus, the higher flux of fatty acids and ketogenesis in LC athletes in the context of overall high energy expenditures could contribute to increased endogenous synthesis of cholesterol by enhancing the cytosolic substrate pool. Increased circulating lathosterol and to a lesser extent desmosterol expressed relative to total cholesterol are markers of de novo cholesterol synthesis. ${ }^{39}$ Lathosterol was lower in LC athletes, indicating that cholesterol overproduction is likely not a major contributor to hypercholesterolaemia. ${ }^{40}$

Campesterol, a marker of exogenous cholesterol absorption, expressed relative to total cholesterol was lower in LC athletes, implying a lower rate of cholesterol absorption may have limited the increase in circulating cholesterol. Serum sitosterol has been demonstrated to positively correlate with cholesterol absorption efficiency ${ }^{41}$ and is higher in endurance athletes, ${ }^{42}$ but was not different in this study. Absolute concentrations of cholestanol were higher in LC athletes, implying decreased conversion of cholesterol to the bile acid chenodeoxycholate. Normally, high cholesterol intake is associated with enhanced biliary cholesterol output to prevent hypercholesterolaemia, ${ }^{43}$ but this mechanism appears to be compromised in LC athletes. The fact that the overall ratio of cholesterol synthesis to absorption markers (ie, the fractional cholesterol balance) was the same between $\mathrm{LC}$ and $\mathrm{HC}$ athletes is consistent with the fact that greater consumption of cholesterol by LC athletes is translated into an expansion of their circulating cholesterol pool. $^{445}$

There may be an unexpected interaction between keto-adaptation and high-volume endurance exercise that manifests in a hypercholesterolaemic phenotype. The LC athletes had been performing relatively high volumes of endurance training for many years. Metabolically, they exhibited a highly refined ability to 

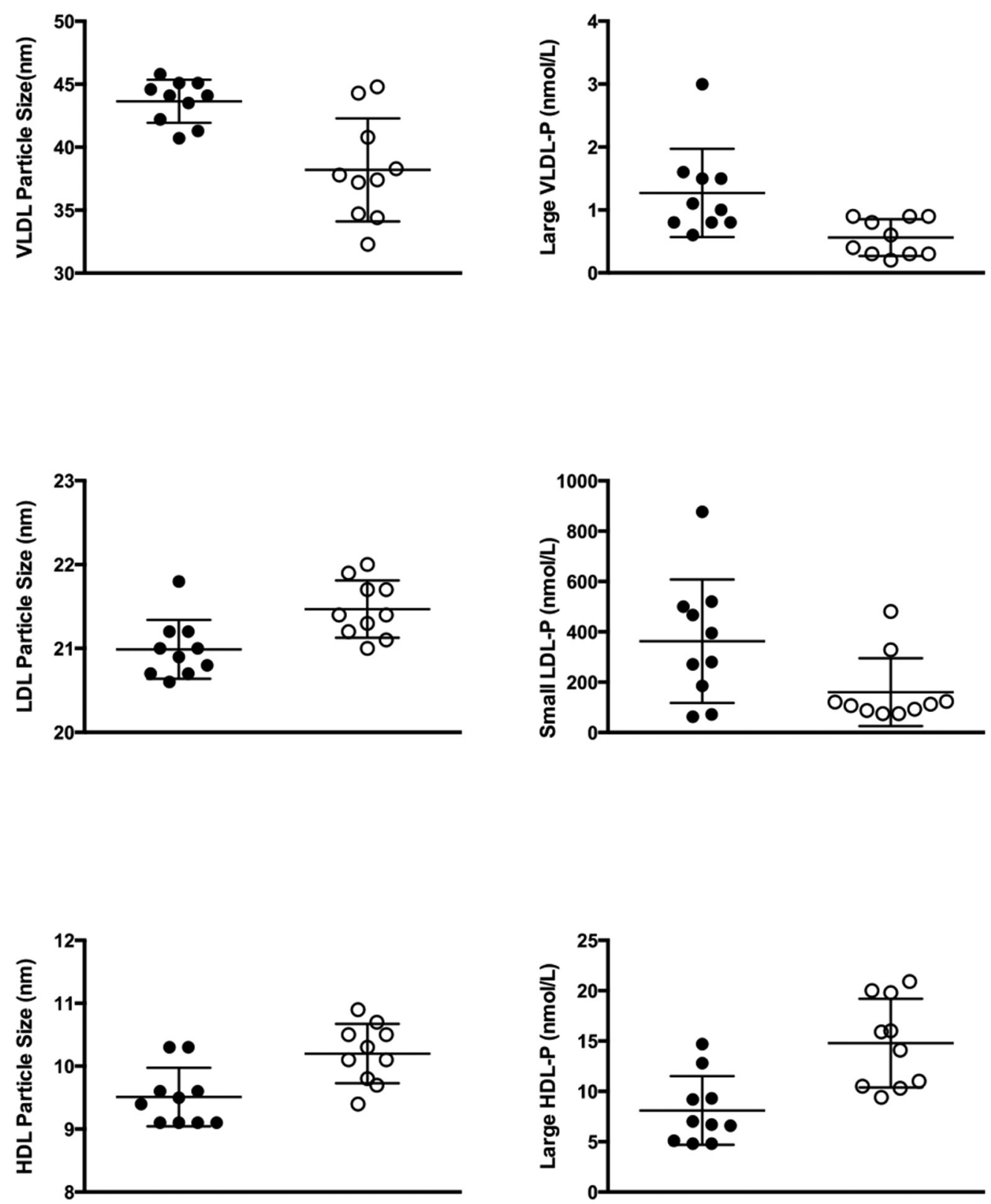

Figure 2 Lipoprotein insulin resistance index measures for high-carbohydrate $(n=10)$ and low-carbohydrate $(n=10)$ ultraendurance athletes. HDL, high-density lipoprotein; HDL-P, HDL particle; LDL, low-density lipoprotein; VLDL-P, very low-density lipoprotein particles.

derive the majority of their energy from lipids at rest and during training. ${ }^{4}$ The substantially greater rates of lipolysis and fatty acid oxidation compared with their HC counterparts may also require adaptations in intravascular lipoprotein metabolism to support the overall greater flux of lipid fuels. Serum HDL-C, specifically HDL2-C, raising effects of exercise are partially attributed to increased expression and activity of skeletal muscle lipoprotein lipase, which breaks down circulating triglycerides, resulting in a transfer of cholesterol and other substances to HDL-C. ${ }^{46}$ It is quite likely keto-adapted athletes increase muscle lipoprotein lipase to enhance use of triglyceride from circulating
VLDL particles. ${ }^{47}$ The greater catabolism of VLDL-TG in LC athletes could result in accumulation of VLDL remnants in the LDL density range. ${ }^{48}$

There are common polymorphisms in successful endurance athletes ${ }^{49}$ that might contribute to a hypercholesterolaemic profile in the context of a ketogenic diet. For example, peroxisome proliferator-activated receptor- $\gamma$ coactivator $1 \alpha$ (PPARGC1A) polymorphisms are related to exceptional endurance capacity ${ }^{50}$ and cholesterol response to dietary fat. ${ }^{51}$ Additional research is necessary to determine the influence of genetic variation as a contributor to the hypercholesterolaemic response to ketogenic diets. 
Table 4 Fractional cholesterol balance and non-cholesterol sterols *

\begin{tabular}{|c|c|c|c|c|c|c|}
\hline & \multicolumn{2}{|c|}{ High-carbohydrate } & \multicolumn{2}{|c|}{ Low-carbohydrate } & \multirow[b]{2}{*}{$P$ values } & \multirow[b]{2}{*}{ Effect size } \\
\hline & Mean & SD & Mean & SD & & \\
\hline Fractional cholesterol balance $†$ & 0.60 & 0.16 & 0.66 & 0.22 & 0.558 & 0.31 \\
\hline \multicolumn{7}{|l|}{ Absolute (mg/L) } \\
\hline Desmosterol & 1.42 & 0.24 & 2.82 & 0.80 & 0.000 & 2.37 \\
\hline Lathosterol & 1.84 & 0.55 & 2.23 & 0.61 & 0.153 & 0.67 \\
\hline Campesterol & 3.5 & 1.46 & 3.86 & 1.37 & 0.577 & 0.25 \\
\hline Sitosterol & 3.07 & 1.03 & 4.34 & 1.75 & 0.064 & 0.88 \\
\hline Cholestanol & 2.23 & 0.44 & 2.8 & 0.43 & 0.009 & 1.31 \\
\hline \multicolumn{7}{|c|}{ Normalised $(10 \dagger \times \mu \mathrm{mol} / \mathrm{mmol}$ of TC) } \\
\hline Desmosterol & 84.8 & 11.55 & 101.9 & 22.35 & 0.046 & 0.96 \\
\hline Lathosterol & 109.4 & 30.67 & 79.6 & 14.41 & 0.016 & 1.24 \\
\hline Campesterol & 195.0 & 59.04 & 134.7 & 43.98 & 0.019 & 1.16 \\
\hline Sitosterol & 167.7 & 44.65 & 145.8 & 55.03 & 0.341 & 0.44 \\
\hline Cholestanol & 132.3 & 24.44 & 101.5 & 13.40 & 0.003 & 1.56 \\
\hline
\end{tabular}

*Values are mean $\pm S D(n=10)$.

†Calculated with normalised values.

TC, total cholesterol.

\section{Clinical relevance}

From a traditional cardiovascular risk perspective, the levels of total and LDL-C observed in LC athletes would classify them as high risk ${ }^{27}$; however, there are conflicting studies on the role of LDL-C and mortality. ${ }^{52}$ A broader look at the lipoprotein profile supports low risk of coronary heart disease and type 2 diabetes. LC athletes had extremely high HDL-C, specifically in the large HDL2-C fraction, which is greater in magnitude than would be expected from a ketogenic diet or exercise training alone. They also exhibited low concentrations of triglycerides and small, dense LDL particles. In over 11000 men and women followed for over a decade, small dense LDL was more strongly associated with an incident of coronary heart disease than traditional blood LDL-C. ${ }^{53}$ The LP-IR derived from the NMR lipoprotein profile correlates with multiple measures of insulin resistance ${ }^{25}$ and is associated with an incident of type 2 diabetes. ${ }^{54}$ The LP-IR was $78 \%$ lower in LC athletes, with absolute scores among the lowest values recorded in over 5000 individuals. $^{25}$

\section{Limitations}

Because of the cross-sectional design of this study, we cannot rule out the possibility that LC athletes were by nature more hypercholesterolaemic or they for some unknown reason self-selected to an LC diet. The relatively small sample size should be expanded to include larger more diverse athletes including women. Since all the measurements made in this study were circulating surrogate markers, it will be important in future work to track athletes for longer periods of time while measuring clinical events and relevant indicators of mortality. None of the participants indicated familial hypercholesterolaemia on their medical questionnaire, but we did not perform genetic testing to test for this condition or other genetic variants. Genotyping in future work would help uncover potential interactions of a ketogenic diet with specific polymorphisms that relate to cholesterol metabolism.

\section{CONCLUSION}

Ketogenic diets in normal-weight and overweight non-athletes result in a moderate increase in total, LDL-C and HDL-C concentrations relative to low-fat diets. $^{28}$ The observation that highly trained, ultra-endurance athletes consuming a very low-carbohydrate/ high-fat diet exhibited a more dramatic and uniform hypercholesterolaemia is counterintuitive since highvolume exercise tends to lower total and LDL-C levels. The explanation for this paradox may involve high intake of cholesterol and saturated fat combined with high-energy demands for lipid metabolism characteristic of the keto-adapted phenotype. LC athletes who adopt a ketogenic diet may experience an expansion of their endogenous cholesterol pool during the adaptation phase of the diet, after which they maintain greater circulating cholesterol levels.

Contributors JSV, WJK, CMM and SDP were involved in the conception and design of the study. All authors made contributions to interpretation of data and drafting the manuscript or revising it critically for important intellectual content.

Funding This work was supported by contributions from Quest Nutrition and The Robert C and Veronica Atkins Foundation.

Competing interests JSV and SDP receive royalties from books on nutrition and exercise. JSV and SDP are founders of Virta Health and have equity positions; BCC is an employee at Virta Health.

Patient consent Obtained. 
Ethics approval The study was approved by The Ohio State University Biomedical IRB.

Provenance and peer review Not commissioned; externally peer reviewed.

Open access This is an open access article distributed in accordance with the Creative Commons Attribution Non Commercial (CC BY-NC 4.0) license, which permits others to distribute, remix, adapt, build upon this work non-commercially, and license their derivative works on different terms, provided the original work is properly cited, appropriate credit is given, any changes made indicated, and the use is non-commercial. See: http://creativecommons.org/licenses/by-nc/4.0/

\section{REFERENCES}

1. Sackner-Bernstein J, Kanter D, Kaul S. Dietary intervention for overweight and obese adults: comparison of low-carbohydrate and low-fat diets. A meta-analysis. PLoS One 2015;10:e0139817.

2. Volek JS, Fernandez ML, Feinman RD, et al. Dietary carbohydrate restriction induces a unique metabolic state positively affecting atherogenic dyslipidemia, fatty acid partitioning, and metabolic syndrome. Prog Lipid Res 2008;47:307-18.

3. Feinman RD, Pogozelski WK, Astrup A, et al. Dietary carbohydrate restriction as the first approach in diabetes management: critical review and evidence base. Nutrition 2015;31:1-13.

4. Volek JS, Freidenreich DJ, Saenz C, et al. Metabolic characteristics of keto-adapted ultra-endurance runners. Metabolism 2016;65:100-10.

5. Volek JS, Noakes T, Phinney SD. Rethinking fat as a fuel for endurance exercise. Eur J Sport Sci 2015;15:13-20.

6. Volek JS, Gómez AL, Kraemer WJ. Fasting lipoprotein and postprandial triacylglycerol responses to a low-carbohydrate diet supplemented with n-3 fatty acids. J Am Coll Nutr 2000;19:383-91.

7. Sharman MJ, Kraemer WJ, Love DM, et al. A ketogenic diet favorably affects serum biomarkers for cardiovascular disease in normal-weight men. J Nutr 2002;132:1879-85.

8. Volek JS, Sharman MJ, Gómez AL, et al. An isoenergetic very low carbohydrate diet improves serum HDL cholesterol and triacylglycerol concentrations, the total cholesterol to HDL cholesterol ratio and postprandial pipemic responses compared with a low fat diet in normal weight, normolipidemic women. J Nutr 2003;133:2756-61.

9. Volek JS, Sharman MJ, Gómez AL, et al. Comparison of a very low-carbohydrate and low-fat diet on fasting lipids, LDL subclasses, insulin resistance, and postprandial lipemic responses in overweight women. J Am Coll Nutr 2004;23:177-84.

10. Volek JS, Phinney SD, Forsythe CE, et al. Carbohydrate restriction has a more favorable impact on the metabolic syndrome than a low fat diet. Lipids 2009;44:297-309.

11. Sharman MJ, Gómez AL, Kraemer WJ, et al. Very low-carbohydrate and low-fat diets affect fasting lipids and postprandial lipemia differently in overweight men. J Nutr 2004;134:880-5.

12. Kodama S, Tanaka S, Saito K, et al. Effect of aerobic exercise training on serum levels of high-density lipoprotein cholesterol: a meta-analysis. Arch Intern Med 2007;167:999-1008.

13. Kelley GA, Kelley KS. Aerobic exercise and HDL2-C: a meta-analysis of randomized controlled trials. Atherosclerosis 2006;184:207-15.

14. Wang $Y, X u D$. Effects of aerobic exercise on lipids and lipoproteins. Lipids Health Dis 2017;16:132.

15. Durstine JL, Grandjean PW, Davis PG, et al. Blood lipid and lipoprotein adaptations to exercise: a quantitative analysis. Sports Med 2001;31:1033-62.

16. Denham J, Nelson CP, O'Brien BJ, et al. Longer leukocyte telomeres are associated with ultra-endurance exercise independent of cardiovascular risk factors. PLoS One 2013;8:e69377.

17. Tomaszewski M, Charchar FJ, Crawford L, et al. Serum C-reactive protein and lipids in ultra-Marathon runners. Am J Cardiol 2004;94:125-6.

18. Phinney SD, Bistrian BR, Wolfe RR, et al. The human metabolic response to chronic ketosis without caloric restriction: physical and biochemical adaptation. Metabolism 1983;32:757-68.

19. Williams PT, Blanche PJ, Rawlings R, et al. Concordant lipoprotein and weight responses to dietary fat change in identical twins with divergent exercise levels 1. Am J Clin Nutr 2005;82:181-7.

20. Warnick GR. Enzymatic methods for quantification of lipoprotein lipids. Methods Enzymol 1986;129:101-23.

21. Warnick GR, Wood PD. National cholesterol education program recommendations for measurement of high-density lipoprotein cholesterol: Executive summary. The national cholesterol education program working group on lipoprotein measurement. Clin Chem 1995;41:1427-33.
22. Friedewald WT, Levy RI, Fredrickson DS. Estimation of the concentration of low-density lipoprotein cholesterol in plasma, without use of the preparative ultracentrifuge. Clin Chem 1972;18:499-502.

23. Otvos JD, Jeyarajah EJ, Bennett DW, et al. Development of a proton nuclear magnetic resonance spectroscopic method for determining plasma lipoprotein concentrations and subspecies distributions from a single, rapid measurement. Clin Chem 1992;38:1632-8.

24. Jeyarajah EJ, Cromwell WC, Otvos JD. Lipoprotein particle analysis by nuclear magnetic resonance spectroscopy. Clin Lab Med 2006;26:847-70.

25. Shalaurova I, Connelly MA, Garvey WT, et al. Lipoprotein insulin resistance index: a lipoprotein particle-derived measure of insulin resistance. Metab Syndr Relat Disord 2014;12:422-9.

26. Matthan NR, Raeini-Sarjaz M, Lichtenstein AH, et al. Deuterium uptake and plasma cholesterol precursor levels correspond as methods for measurement of endogenous cholesterol synthesis in hypercholesterolemic women. Lipids 2000;35:1037-44.

27. National cholesterol education program (ncep) expert panel on detection, evaluation, and treatment of high blood cholesterol in adults (adult treatment panel iii). Third report of the national cholesterol education program (ncep) expert panel on detection, evaluation, and treatment of high blood cholesterol in (Adult Treatment Panel III) final report. Circulation 2002;106:3143-421.

28. Volek JS, Sharman MJ, Forsythe CE. Modification of lipoproteins by very low-carbohydrate diets. J Nutr 2005;135:1339-42.

29. Hespanhol Junior LC, Pillay JD, van Mechelen W. Meta-analyses of the effects of habitual running on indices of health in physically inactive adults. Sports Med 2015;45:1455-68.

30. Brown RC, Cox CM. Effects of high fat versus high carbohydrate diets on plasma lipids and lipoproteins in endurance athletes. Med Sci Sports Exerc 1998;30:1677-83.

31. Thompson PD, Cullinane EM, Eshleman R, et al. The effects of highcarbohydrate and high-fat diets on the serum lipid and lipoprotein concentrations of endurance athletes. Metabolism 1984;33:1003-10.

32. Krauss RM. Dietary and genetic probes of atherogenic dyslipidemia. Arterioscler Thromb Vasc Biol 2005;25:2265-72.

33. Volk BM, Kunces LJ, Freidenreich DJ, et al. Effects of step-wise increases in dietary carbohydrate on circulating saturated Fatty acids and palmitoleic Acid in adults with metabolic syndrome. PLoS One 2014;9:e113605.

34. Clarke R, Frost C, Collins R, et al. Dietary lipids and blood cholesterol: quantitative meta-analysis of metabolic ward studies. BMJ 1997;314:112-7.

35. Berger S, Raman G, Vishwanathan R, et al. Dietary cholesterol and cardiovascular disease: a systematic review and meta-analysis. Am J Clin Nutr 2015;102:276-94.

36. Wei ZH, Wang H, Chen XY, et al. Time- and dose-dependent effect of psyllium on serum lipids in mild-to-moderate hypercholesterolemia: a meta-analysis of controlled clinical trials. Eur J Clin Nutr 2009;63:821-7.

37. Robinson AM, Williamson DH. Physiological roles of ketone bodies as substrates and signals in mammalian tissues. Physiol Rev 1980;60:143-87.

38. Endemann G, Goetz PG, Edmond J, et al. Lipogenesis from ketone bodies in the isolated perfused rat liver. Evidence for the cytosolic activation of acetoacetate. J Biol Chem 1982;257:3434-40.

39. Kempen HJ, Glatz JF, Gevers Leuven JA, et al. Serum lathosterol concentration is an indicator of whole-body cholesterol synthesis in humans. J Lipid Res 1988;29:1149-55.

40. Björkhem I, Miettinen T, Reihnér E, et al. Correlation between serum levels of some cholesterol precursors and activity of HMG-CoA reductase in human liver. J Lipid Res 1987;28:1137-43.

41. Tilvis RS, Miettinen TA. Serum plant sterols and their relation to cholesterol absorption. Am J Clin Nutr 1986;43:92-7.

42. Sutherland WH, Robertson MC, Williamson SA, et al. Plasma noncholesterol sterols in male distance runners and sedentary men. Eur J Appl Physiol Occup Physiol 1991;63:119-23.

43. Quintão E, Grundy SM, Ahrens EH. Effects of dietary cholesterol on the regulation of total body cholesterol in man. $J$ Lipid Res 1971;12:233-47.

44. Katan MB, Beynen AC. Characteristics of human hypo- and hyperresponders to dietary cholesterol. Am J Epidemiol 1987;125:387-99.

45. Nestel PJ, Poyser A. Changes in cholesterol synthesis and excretion when cholesterol intake is increased. Metabolism 1976;25:1591-9.

46. Blazek A, Rutsky J, Osei K, et al. Exercise-mediated changes in high-density lipoprotein: impact on form and function. Am Heart $J$ 2013;166:392-400

47. Helge JW, Watt PW, Richter EA, et al. Fat utilization during exercise: adaptation to a fat-rich diet increases utilization of plasma fatty 
acids and very low density lipoprotein-triacylglycerol in humans. $J$ Physiol 2001;537(Pt 3):1009-20.

48. Kuusi T, Kostiainen E, Vartiainen $\mathrm{E}$, et al. Acute effects of marathon running on levels of serum lipoproteins and androgenic hormones in healthy males. Metabolism 1984;33:527-31.

49. Williams AG, Folland JP. Similarity of polygenic profiles limits the potential for elite human physical performance. J Physiol 2008;586:113-21.

50. Lucia A, Gómez-Gallego F, Barroso I, et al. PPARGC1A genotype (Gly482Ser) predicts exceptional endurance capacity in European men. J Appl Physiol 2005;99:344-8.

51. Schmoldt A, Benthe HF, Haberland G. Digitoxin metabolism by rat liver microsomes. Biochem Pharmacol 1975;24:1639-41.
52. Ravnskov U, Diamond DM, Hama R, et al. Lack of an association or an inverse association between low-density-lipoprotein cholesterol and mortality in the elderly: a systematic review. BMJ Open 2016;6:e010401.

53. Hoogeveen RC, Gaubatz JW, Sun W, et al. Small dense low-density lipoprotein-cholesterol concentrations predict risk for coronary heart disease: the Atherosclerosis Risk In Communities (ARIC) study. Arterioscler Thromb Vasc Biol 2014;34:1069-77.

54. Harada PHN, Demler OV, Dugani SB, et al. Lipoprotein insulin resistance score and risk of incident diabetes during extended follow-up of 20 years: The Women's Health Study. J Clin Lipidol 2017;11:1257-67. 